Projets

de paysage

\section{Projets de paysage}

Revue scientifique sur la conception et l'aménagement de l'espace

24 | 2021

Le paysage au prisme du politique

\title{
Quand le paysage ouvre un horizon politique : Aix- Marseille-Provence métropole et le sentier métropolitain GR2013
}

When the Landscape Provides a Political Perspective: The Aix-Marseille-

Provence Metropolis and the GR2013 Metropolitan Trail

\section{Sylvie Salles}

\section{OpenEdition}

\section{Journals}

Édition électronique

URL : https://journals.openedition.org/paysage/20430

DOI : $10.4000 /$ paysage. 20430

ISSN : 1969-6124

Éditeur :

École nationale supérieure du paysage de Versailles-Marseille, Institut national des sciences appliquées Centre Val de Loire - École de la nature et du paysage, École nationale supérieure d'architecture et de paysage de Bordeaux, École nationale supérieure d'architecture et de paysage de Lille, Agrocampus Angers

\section{Référence électronique}

Sylvie Salles, «Quand le paysage ouvre un horizon politique : Aix-Marseille-Provence métropole et le sentier métropolitain GR2013 », Projets de paysage [En ligne], 24 | 2021, mis en ligne le 20 septembre 2021, consulté le 01 octobre 2021. URL : http://journals.openedition.org/paysage/20430 ; DOI https://doi.org/10.4000/paysage.20430

Ce document a été généré automatiquement le 1 octobre 2021.

La revue Projets de paysage est mise à disposition selon les termes de la Licence Creative Commons Attribution - Pas d'Utilisation Commerciale - Pas de Modification 4.0 International. 


\title{
Quand le paysage ouvre un horizon politique : Aix-Marseille-Provence métropole et le sentier métropolitain GR2013
}

\author{
When the Landscape Provides a Political Perspective: The Aix-Marseille- \\ Provence Metropolis and the GR2013 Metropolitan Trail
}

Sylvie Salles

Cet article éclaire le paysage au prisme du politique au travers de l'analyse d'un horizon politique commun pour la métropole Aix-Marseille-Provence (AMP) qui s'est ouvert avec le projet touristique, culturel et artistique du sentier métropolitain de grande randonnée GR2013. Le paysage convoqué ici renvoie à la définition relationnelle, sensible et symbolique des sociétés à leurs environnements (Besse, 2013), retenue pour ce numéro de Projets de paysage. Plus spécifiquement, cette relation s'appuie sur une expérience in situ des paysages de la métropole dévoilée et partagée dans la marche par les artistes et les guides du sentier (Tiberghien, 2004 ; Lanaspèze et Lavessière, 2020). Le prisme du politique a une double acception. La dimension collective et culturelle de la création et de l'animation du GR2013 est une forme d'organisation d'un vécu commun, qui s'attache à des territoires réunis dans une métropole institutionnelle, dont la création avait été actée dès janvier $2014^{1}$. De fait, ce monde commun, autour des projets accueillis sur le GR2013, n'est jamais réellement dissociable d'une mise en commun en quête d'un récit collectif autour d'un paysage métropolitain vu comme un objet politique fédérateur (Di Méo, 2010). La convergence entre ces deux événements permet de questionner la distinction que John Brinckerhoff Jackson opère entre un paysage vernaculaire, produit par l'usage et l'adaptation des hommes à leur milieu de vie, et un paysage politique, renvoyant à des organisations globalisantes, qu'il décrit comme deux modalités paysagères concurrentes qui coexistent (Jackson, 2003). 
2 Le projet du GR2013, créé pour Marseille-Provence capitale européenne 2013 de la culture, a participé à la promotion territoriale de la Provence orchestrée par la chambre de commerce et d'industrie pour anticiper la création de la métropole. Ici, le sentier métropolitain va donner une forme concrète à une métropole essentiellement défendue par la société civile. Toutefois, en rendant visible le territoire multipolarisé de l'aire urbaine de Marseille et d'Aix-en-Provence dans lequel chacun vit et travaille, le GR2013 a aussi matérialisé des paysages habités, produits par un vécu métropolitain plus trivial. Le paysage vernaculaire des périphéries de la ville contemporaine se glisse au milieu d'un paysage politique produit par des choix urbanistiques. Dans la métropole Aix-Marseille-Provence, ce paysage périurbain est toujours en interaction avec des milieux naturels particulièrement riches écologiquement, voire il s'est construit dedans. Ainsi, le paysage que le sentier métropolitain révèle est le témoin d'un brouillage complexe, où un urbanisme peu régulé a fabriqué des recouvrements entre paysages vernaculaires et politiques, mais aussi entre dynamiques naturelles et culturelles. Derrière ces recouvrements, il y a des "pays-paysages » décrits par l'historien René Borruey : « le verdoyant pays d'Aix, le bleu pays de l'étang de Berre, le plat pays de Fos et le grand pays de Marseille contenant à lui seul ville, mer, campagne et montagnes » (Borruey, 2018, p. 22). Il y a aussi le fait que ces pays-paysages sont à la fois des entités géomorphologiques et des organisations territoriales, puisque les massifs ont délimité des territorialités éclatées, dans lesquelles s'est glissée une structuration institutionnelle tout aussi éclatée (Roncayolo, 2014). Il ne s'agit pas d'un point de vue déterministe, mais d'une structuration qui met en avant des interférences entre les modalités, vernaculaires et politiques, de production de paysages qui ici sont aussi des pays-paysages. Cela élargit et spécifie la part relationnelle du paysage dans sa dimension politique qui, dans ce territoire méditerranéen encore plus qu'ailleurs, renvoie à des dynamiques anthropiques et naturelles jamais réellement dissociées.

Cette réflexion s'adosse aux résultats de la recherche Popsu métropole $\mathrm{AMP}^{2}$, qui interroge la manière dont le paysage, mis au cœur de la construction métropolitaine, est aussi acteur d'une fabrique plus quotidienne de ses territoires. Cet article, s'appuyant sur les discours des marcheurs à l'origine du tracé du GR2013 et d'acteurs impliqués dans la construction de la métropole, se focalise sur la manière dont l'expérience in situ des paysages depuis le GR2013 a fait émerger une vision métropolitaine incarnée dans une proximité quotidienne à un paysage déjà reconnu pour sa valeur culturelle et touristique. Le paysage est depuis le GR2013 une « pratique culturelle » dans un contexte où, du côté de la construction métropolitaine, il est aussi un « instrument de pouvoir culturel » (Mitchell, 2002), mais sans visée impérialiste. En effet, nous y avons plutôt vu des mises en écho de représentations différentes du paysage. Qu'elles soient portées par des projets artistiques et culturels ou par des visions métropolitaines intégratrices, ces formes d'attention au paysage sont confrontées à un sens commun lié à la double valeur, écologique et paysagère, de milieux habités qui sont également des paysages méditerranéens emblématiques. Ici, depuis le GR2013, le sens réel du paysage, comme le définit Kenneth R. Olwig, renvoie à un paysage politique qui est aussi un lieu habité et un espace d'interactions environnementales (Olwig, 1996, p.630). C'est ce processus d'émergence d'un sens contemporain, à la fois vernaculaire et politique, du paysage d'Aix-Marseille-Provence que le sentier métropolitain et ses projets activent en proposant des représentations que William J. T. Mitchell qualifie de « secondaires ». En effet, les paysages, auxquels le 
GR2013 donne accès, sont eux-mêmes les médiums des représentations culturelles et politiques qui les ont produits.

«Landscape may be represented by painting, drawing, or engraving; by photography, film, and theatrical scenery; by writing, speech, and presumably even music and other "Sound image". Before all these secondary representations, however, landscape is itself a physical and multisensory medium (earth, stone, vegetation, water, sky, sound and silence, light and darkness, etc.) in which cultural meaning and values are encoded, whether they are put thereby the physical transformation of a place in landscape gardening or architecture, or found in a place formed, as we say, "by nature"3.» (Mitchell, 2002, p. 14.)

Quel paysage le sentier dessine-t-il ou (ré)invente-t-il ? En quoi et comment contribue$\mathrm{t}$-il à redéfinir les frontières entre paysage vernaculaire et paysage politique, $a$ fortiori en pleine construction politique d'une métropole? Pour comprendre ces interférences et ces glissements, dans un premier temps nous analyserons la spécificité de ce sentier métropolitain et du projet qu'il porte, en regard des autres sentiers métropolitains promus par les mêmes concepteurs. Puis, nous nous attacherons aux figures contemporaines du paysage vernaculaire, à travers les représentations culturelles et politiques que certains des projets partenaires du GR2013 font émerger. Enfin, nous verrons comment, en retour, le récit du paysage comme horizon métropolitain commun, glisse vers un paysage métropolitain qui est un bien commun relevant d'usages et de modes de gestion partagés (Sgard, 2010) dans les derniers projets du Bureau des guides du GR2013.

Figure 1. Les paysages de la métropole Aix-Marseille-Provence depuis le GR2013

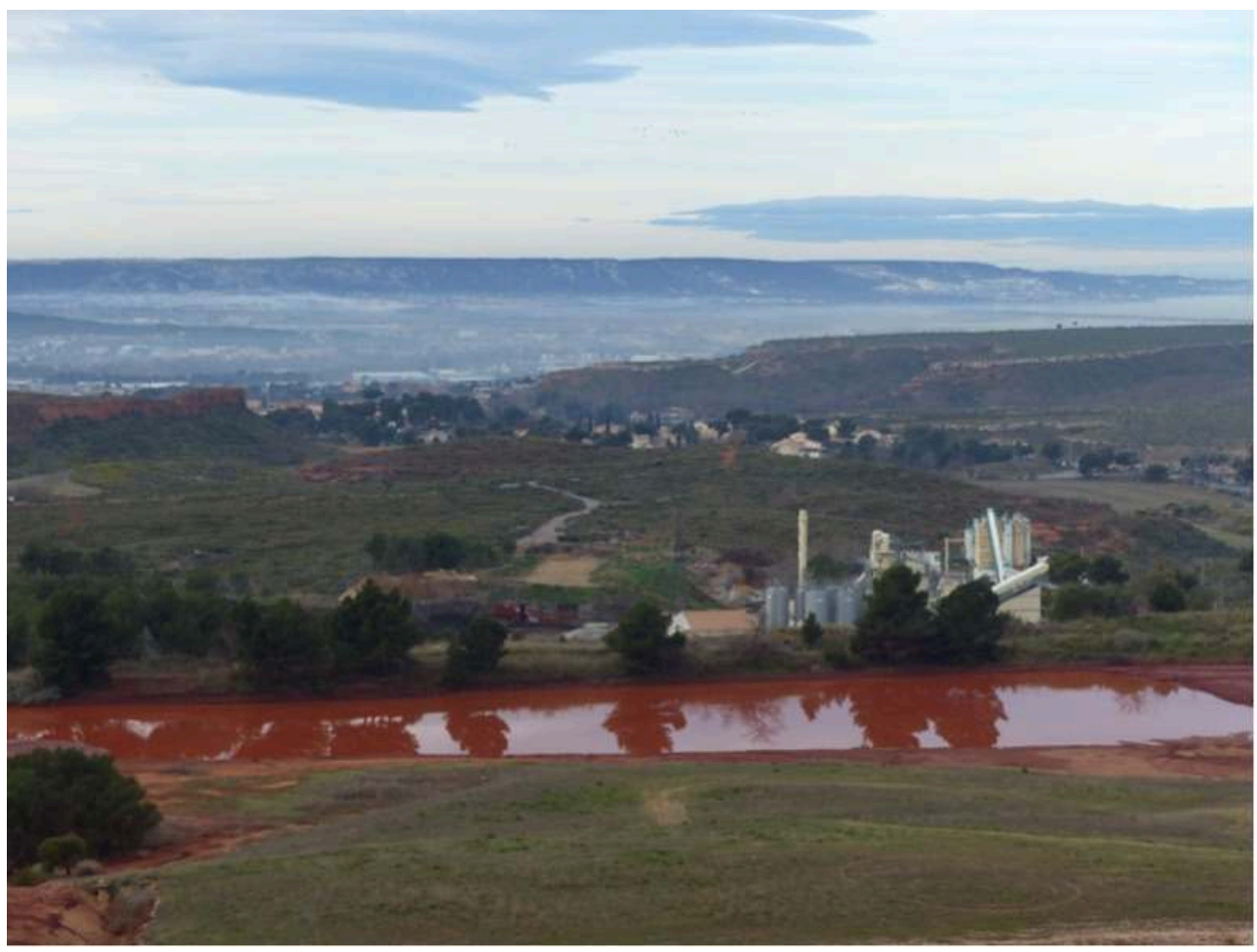

Source : Sylvie Salles, 2021. 


\section{Le sentier métropolitain GR2013 : dessiner une métropole par la marche}

5 Le GR2013 est le premier sentier métropolitain de grande randonnée, créé à l'initiative de l'éditeur marseillais Baptiste Lanaspèze, qui a cofondé en 2014 le Bureau des guides du GR2013, pour continuer à faire vivre le sentier, ainsi que l'Agence des sentiers métropolitains, maître d'œuvre d'autres sentiers métropolitains, dont celui du Grand Paris, et qui anime le réseau Metropolitan Trails ${ }^{4}$.

\section{Le tracé du GR2013 aux frottements de la ville et de la nature}

Le GR2013 est au départ une commande du directeur de l'association chargée de l'organisation de Marseille-Provence capitale européenne 2013 de la culture, Bernard Latarjet, pour réaliser un projet illustrant le rapport ville-nature propre à Marseille que Baptiste Lanaspèze avait décrit dans Marseille, ville sauvage :

«À la fois industrielle et rurale, en friche et bétonnée, Marseille est un laboratoire à ciel ouvert où se réinvente la relation entre aire urbaine et nature, entre sauvage et civilisé, entre nord et sud. Le génie de la ville bouscule et interroge l'écologie urbaine, ce champ de recherche émergent qui, entre écologie et sociologie, propose de nouveaux modèles pour les cités de demain. » (Lanaspèze, 2020, p. 1).

S'il n'évoque pas directement le paysage, pour lui la création du Parc national des Calanques, effective en 2012, caractérise de façon exemplaire ce lien en reconnaissant la valeur culturelle et écologique d'un massif périurbain. Le rapprochement qu'il fait entre ville et nature réinterprète des liens fondateurs entre l'homme et cet environnement méditerranéen, où la maîtrise de l'eau a garanti l'habitabilité et la fertilité du territoire. Même s'ils ont été mis à mal par des décennies de développements urbains, ces liens restent perceptibles dans une proximité entre l'homme et la nature où « il est impossible de parler de paysage sans parler de jardins et [de] parler de la campagne sans évoquer la ville proche» (Tamisier, 1995, p. 39). Aujourd'hui, cette relation se devine dans le jardinage de délaissés, dans l'appropriation d'espaces naturels comme espaces de proximité, dans la pratique quotidienne d'espaces ouverts, jardinés, agricoles ou naturels.

Dans l'aire urbaine de Marseille, cette «ville-nature» est une forme urbaine où s'interpénètrent de grands éléments de paysages naturels, des espaces construits et des pratiques urbaines (Chalas, 2010). Les interpénétrations y sont particulièrement nombreuses.

«Transformée ou sanctuarisée, cultivée ou protégée, vécue ou rêvée, arpentée ou ignorée, en friche ou en jardin, publique ou privée, vaste ou minuscule, délaissée ou domestiquée, déblayée ou remblayée, bâtie ou jardinée : la nature prend des formes multiples dans le territoire urbain marseillais et toutes ces interactions entre ville et nature sont riches d'implications sociologiques. » (Lanaspèze, 2020, p. 36.)

Ces formes résultent des modalités relationnelles de production du paysage vernaculaire. De la même manière, aux marges des développements périurbains, industriels ou autoroutiers de l'aire métropolitaine, le tracé du GR2013 rend visibles des interpénétrations ville-nature plus frontales où les paysages de l'aménagement du territoire sont aussi des paysages appropriés. De ce point de vue, la création du sentier métropolitain est un acte culturel, non parce qu'il est lié à Marseille-Provence capitale européenne 2013 de la culture, mais parce qu'il a été conçu par Baptiste Lanaspèze et 
Nicolas Mémain - qui se définit lui-même en poète urbain passionné d'urbanisme comme un objet métropolitain low tech afin de faire évoluer les représentations des paysages de la métropole et de la métropole elle-même.

\section{Un projet culturel pour la métropole}

8 Le parti pris des concepteurs du sentier de grande randonnée et du collectif de marcheurs qui l'ont repéré sur le terrain a été de faire éprouver l'ampleur, la beauté et la complexité des territoires urbanisés où les métropolitains habitent. Le projet s'insère dans un impensé de la construction métropolitaine, dénoncé par l'urbaniste Simon Ronai qui a montré que les représentations de l'aire métropolitaine, basées sur des images emblématiques de la Provence (la Méditerranée, la Sainte-Victoire de Cézanne, la Sainte-Baume de Pagnol, etc.), étaient en décalage complet avec la manière dont le territoire fonctionne et est vécu. Le lien qui est fait entre identité et attractivité est pour lui contreproductif car il accentue les oppositions littoral/arrière-pays, Méditerranée/Provence, ville/nature qui fondent l'éclatement du territoire (Ronai, 2009 p. 140). Au contraire, le GR2013 met en avant un imaginaire métropolitain bien plus banal. Cet imaginaire "sécrété par la société et fait d'observations et d'expériences » est, pour le géographe Marcel Roncayolo, bien plus sérieux que l'image que l'on vend aux "étrangers", puisqu'il est capable d'en imposer à ces images externes et d'amorcer des changements depuis l'intérieur des territoires (Roncayolo, 2014, p. 296). C'est le pari qu'a fait Baptiste Lanaspèze, avec l'idée de renouveler les représentations des paysages traversés, en référence aux paysages ordinaires des "News Topographics ${ }^{5}$ ( Salvesen, 2010) et de la mission photographique de la Datar ${ }^{6}$ (Bertho, 2013). Le sentier métropolitain GR2013 permet d'entrer dans cet intérieur, peu connu et peu reconnu, de la métropole. Il amorce une "artialisation in situ " (Roger, 1997), qui est ici un processus d'ouverture d'un nouvel imaginaire métropolitain. En effet, les paysages vernaculaires et politiques que le sentier traverse sont aussi les paysages produits par l'éclatement territorial que la construction métropolitaine cherche à enrayer. 
Figure 2. Carte des lieux de Marseille-Provence 2013

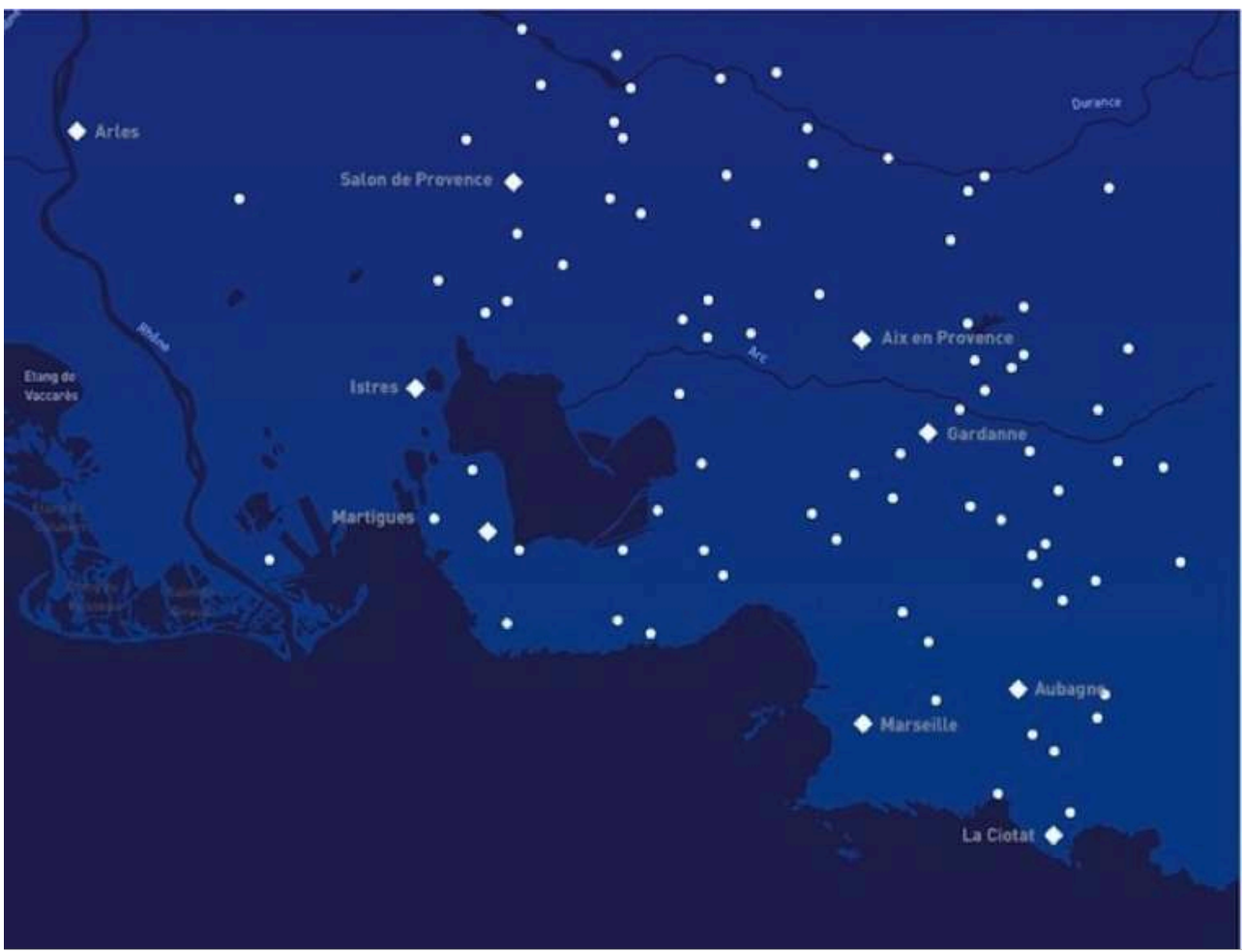

Source : MP2013.

Les $675 \mathrm{~km}$ de sentier du GR2013 ont été repérés et tracés, à partir de 2011, par un collectif d'artistes et de marcheurs qui ont arpenté l'aire métropolitaine à la recherche de chemins non bitumés, en passant sous les autoroutes, en suivant des voies de chemin de fer désaffectées, en traversant d'anciens sites industriels ou en longeant des périphéries industrielles et commerciales. L'idée était de donner accès à des espaces agricoles, aux vallées des fleuves côtiers, aux collines qui jouxtent la ville, etc. Ce chemin de traverse ordonne la lecture d'un territoire métropolitain en cours de structuration dans une figure qui, parce qu'elle est facilement lisible, devient fédératrice. La double boucle (autour de l'étang de Berre, à l'ouest, et autour des massifs de l'Étoile, au nord, et du Garlaban, au nord-est) se croise à la gare d'Aix TGV. Nicolas Mémain en a eu l'idée pour donner une forme simple et reconnaissable à un territoire métropolitain difficile à représenter. Le sentier métropolitain participe ainsi d'une dynamique, qu'Augustin Berque appelle " empreinte-matrice » et dans laquelle « la société perçoit son milieu en fonction de l'usage qu'elle en fait; réciproquement, elle l'utilise en fonction de la perception qu'elle en a. Des matrices phénoménologiques (les schèmes de perception et d'interprétation du milieu) ne cessent ainsi d'engendrer des empreintes physiques (les modes d'aménagement du milieu) ; lesquels, à leur tour, influent ces matrices, et ainsi de suite » (Berque, 1990, p. 44). Le paysage, depuis le sentier, est le témoin et le motif de ces évolutions ; à la fois empreinte et matrice d'une culture en train de se construire comme métropolitaine. Les artistes-marcheurs du GR2013 sont des agents de ce processus, au sens où ils s'inscrivent eux-mêmes dans le jeu politique de la construction métropolitaine, tout en ayant le souci de formuler un projet qui leur soit propre. 
Figure 3. Carte du GR2013

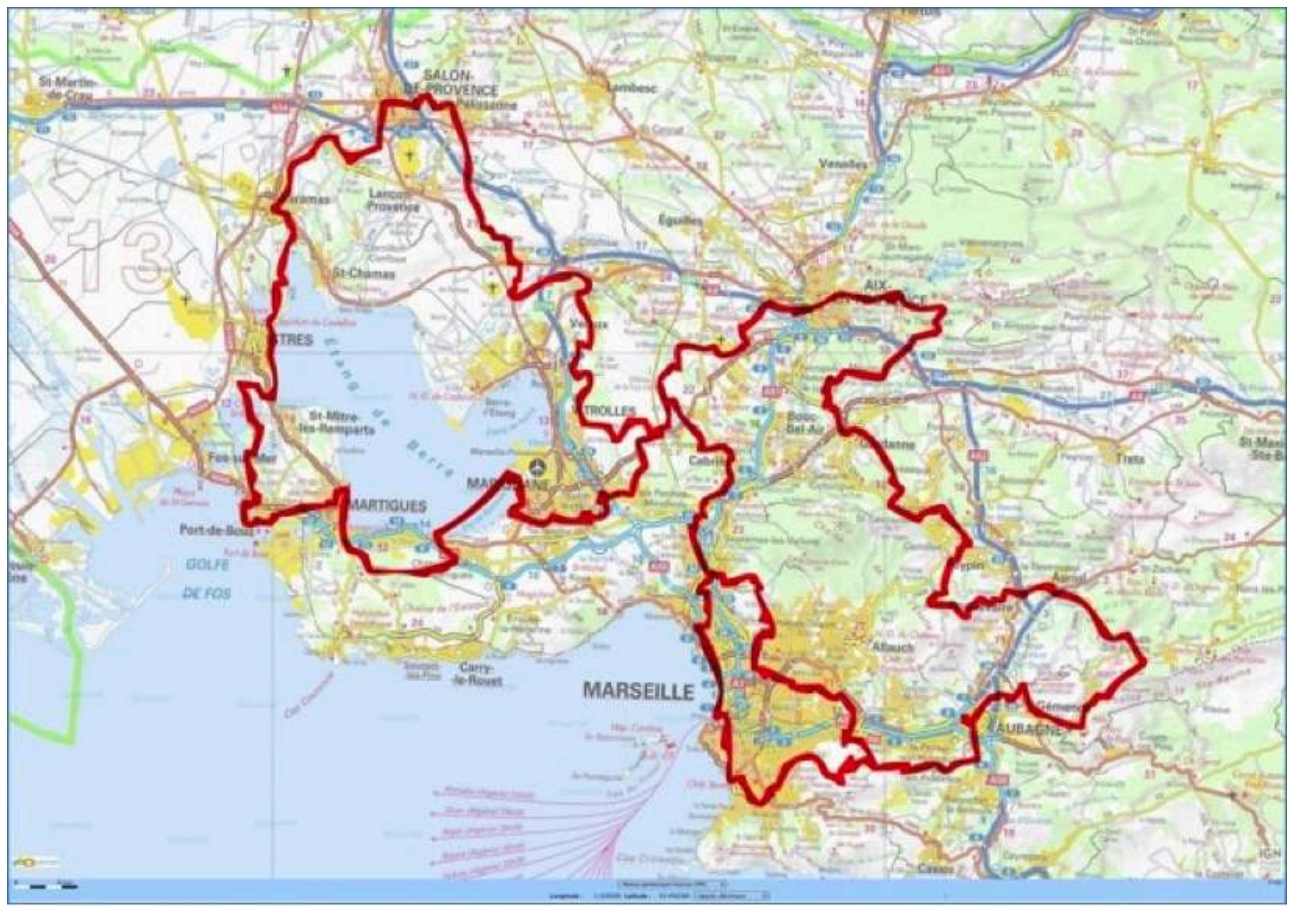

Source : IGN

\section{Le Sentier métropolitain, un label qui se diffuse}

Leur projet s'est précisé et stabilisé plus tard, avec la diffusion du modèle labellisé des Sentiers métropolitains, via la rédaction d'une charte et la création du réseau des Sentiers métropolitains, à l'occasion de l'exposition "Connectivités ", en 2017 au Mucem (Marseille). La charte précise qu'un «Sentier métropolitain est un sentier de randonnée pédestre en milieu urbain ou périurbain, qui permet d'explorer une métropole ou une aire métropolitaine en plusieurs jours ». Cet objectif de connaissance des métropoles contemporaines revient sur l'héritage des premiers marcheurs urbains, en affirmant le rôle de l'expérience du terrain au contact de la rugosité des banlieues, autoroutes, aéroports, pavillons, zones industrielles, friches ou fragments de campagnes. Les marcheurs du réseau des Sentiers métropolitains suivent tous les pas de pionniers. C'étaient des étudiants architectes à la recherche d'une culture du réel qui, au début des années 1990, investissaient par une action artistique ou sociale des espaces urbains et périurbains. Le laboratoire d'art urbain Stalker ${ }^{7}$ a inauguré une pratique de la marche dans Rome, en traversant une "ville parallèle » composée de lieux résiduels exclus par l'urbanisme, mais dotée de structures propres, de réseaux de relations et d'habitants (Stalker, 2000; Careri, 2013). Les membres de Stalker ont découvert, cartographié et donné de l'attention à des lieux oubliés, à leurs mémoires, aux liens sociaux qui s'y installent. Au même moment à Bordeaux, le collectif de création urbaine Bruit du frigo, créé par Gabi Farage et Yvan Detraz ${ }^{8}$, réunit des « défricheurs d'espaces » et des « activateurs d'usages " pour générer des urbanités à partir de la manière dont les gens vivent. Ils ont conduit des ateliers d'urbanisme, construit des refuges urbains et organisé des explorations périurbaines avec les habitants. Pour son diplôme d'architecte, Yvan Destraz part en randonnée à travers la 
périphérie de Bordeaux pour repérer des délaissés périurbains pouvant devenir des "terres communes». Il n'a pas pensé à en faire un sentier de randonnée, alors que l'esprit des Sentiers métropolitains est déjà là (Detraz, 2020). La pratique de la marche urbaine se diffuse progressivement, via le blog banlieuedeparis ${ }^{9}$ ou le Mouvement des chemineurs de l'École nationale supérieure d'architecture de Versailles où l'on retrouve le cofondateur du Bureau des guides du GR2013 Alexandre Field. En revanche, à la différence de ces pionniers, le modèle des Sentiers métropolitains affiche aujourd'hui un positionnement clairement opérationnel à la croisée de l'aménagement, de l'art, du tourisme et de l'écologie.

11 Le projet du Sentier métropolitain du (Très) Grand Paris, dernier né en filiation directe avec le GR2013, montre bien ces évolutions. Il est revendiqué comme un acte d'écologie politique par ses fondateurs qui sont toujours Baptiste Lanaspèze et Alexandre Field, cette fois avec Paul-Hervé Lavessière, Jens Denissen et Denis Moreau ${ }^{10}$. Cette écologie «parle de la façon dont les hommes et les femmes occupent, soignent et s'approprient leurs terres et révèle les sédimentations historiques et les négociations qui façonnent les milieux de vie des métropolitains ${ }^{11}$ ». En même temps, ils décrivent le sentier comme le plus grand espace public d'île de France, à la fois une infrastructure touristique, un équipement pédestre géant et un centre d'art que financent les principaux acteurs institutionnels de la métropole du Grand Paris et de la région Île de France. Son tracé a été médiatisé lors de l'inauguration de l'exposition «L'art des Sentiers métropolitains " mi-juillet 2020 au Pavillon de l'Arsenal (Paris). Ses commissaires, Baptiste Lanaspèze et Paul-Hervé Lavessière, décrivent le projet de sentier comme une « machine à changer Paris » en créant une communauté symbolique de dimension métropolitaine. Le récit fédérateur du Sentier métropolitain du (Très) Grand Paris met en regard, sans pour l'instant les faire dialoguer, le «paysage politique " des grands travaux de l'État et la rugosité d'un "paysage vernaculaire » périurbain qui reste à identifier sur le terrain ${ }^{12}$. Toutefois, cette référence n'est pas explicite. Les concepteurs convoquent d'un côté des plans ou des schémas directeurs urbanistiques et, de l'autre, une expérience du terrain par la marche encore à ancrer dans l'épaisseur culturelle des lieux traversés. Ce détour par le Grand Paris est important pour éclairer la tension que les Sentiers métropolitains entretiennent entre le récit fédérateur d'un cadre métropolitain institutionnel et l'attention au subjectif propre aux projets artistiques et culturels. Ce détour permet notamment d'identifier des points de vigilance dans ce glissement entre des visions politiques et culturelles d'un même paysage. En revanche, ce sentier encore en projet n'offre pas suffisamment de recul pour identifier les interférences que nous cherchons à éclairer dans la mise en relation, par un projet culturel de sentier, de représentations où politique et vernaculaire se rencontrent sur un même territoire.

\section{Les figures de la fabrication d'un paysage métropolitain depuis le GR2013}

12 À la différence du Sentier du Grand Paris, le récit que le Sentier métropolitain GR2013 véhicule, autour des contacts et des frottements entre la ville et la nature, a une épaisseur sociale et culturelle qui fait particulièrement sens pour le territoire d'AixMarseille-Provence. 


\section{Une constellation associative autour de la randonnée et de la marche}

Ce récit incarne le sens culturel d'un paysage lié à une habitabilité construite sur le temps long (Tamisier, 1995), dont le potentiel d'actualisation ne se limite pas aux figures contemporaines de «l'entre-ville» (Sieverts, 2004) ou de la « co-extensivité de la ville et du territoire » (Chalas, 2010). En effet, les chercheurs du Centre d'études, de recherches et de formation institutionnelle du Sud-Est (Cerfise ${ }^{13}$ ) ont montré que Marseille était une ville d'invention d'une nature qui était un lieu d'enjeux économiques, sociaux, écologiques et de pouvoir (Peraldi et al., 1988). Les clubs d'excursion ont eu un rôle initiateur, en installant par la randonnée une familiarité à la nature des périphéries d'Aix et de Marseille. Si les antennes locales du Club alpin français ou du Touring Club de France étaient plus élitistes, la Société des excursionnistes marseillais ou "excurs", fondée par un libraire, a pris une place prépondérante en organisant des excursions ouvertes à tous et en publiant des petits guides d'excursions aux environs de Marseille. Michel Peraldi et Jean-Louis Parisis ont analysé cette société d'un point de vue micropolitique, au sens de Gilles Deleuze et Félix Guattari, c'est-à-dire en s'intéressant à des pouvoirs disséminés dans des activités sociales ou quotidiennes qui ont capacité à instituer une pratique sociale (Deleuze et Guattari 1980). Ce qu'ils disent sur les excurs résonne mot pour mot avec la marche d'exploration que le GR2013 a réactivée et réactualisée. Les guides excursionnistes, comme ceux du GR2013, sont des experts, ayant une connaissance quasi scientifique des massifs et des collines d'Aix et de Marseille que tout le monde pense connaître, et des passeurs, engageant un nouveau mode de territorialisation. Michel Peraldi et JeanLouis Parisis ont montré que les excursionnistes, en instituant une nouvelle pratique sociale - la randonnée de proximité -, ont aussi dessiné des agencements villecampagne ou ville-nature inédits. En ce sens, au fil des randonnées, les excursionnistes ont préparé l'aménagement du territoire.

«Position transitionnelle des excurs, embarqués sans qu'ils le sachent vraiment

dans l'émergence de nouveaux ordres sociaux et territoriaux, attelés sur les lieux de nature à la fabrication de nouvelles utilités sociales " (Peraldi et Parisis 1981, p. 51).

Ces nouveaux ordres sociaux et territoriaux ont été d'autant plus opérants, au moment où des mutations économiques et sociales redéfinissaient le rapport de Marseille à son arrière-pays. De ce point de vue, en tant qu'intermédiaires culturels, les excursionnistes ont contribué à réarticuler des modes de production vernaculaires et politiques des paysages. 
Figure 4. Le massif de l'Arbois, espace naturel classé de proximité

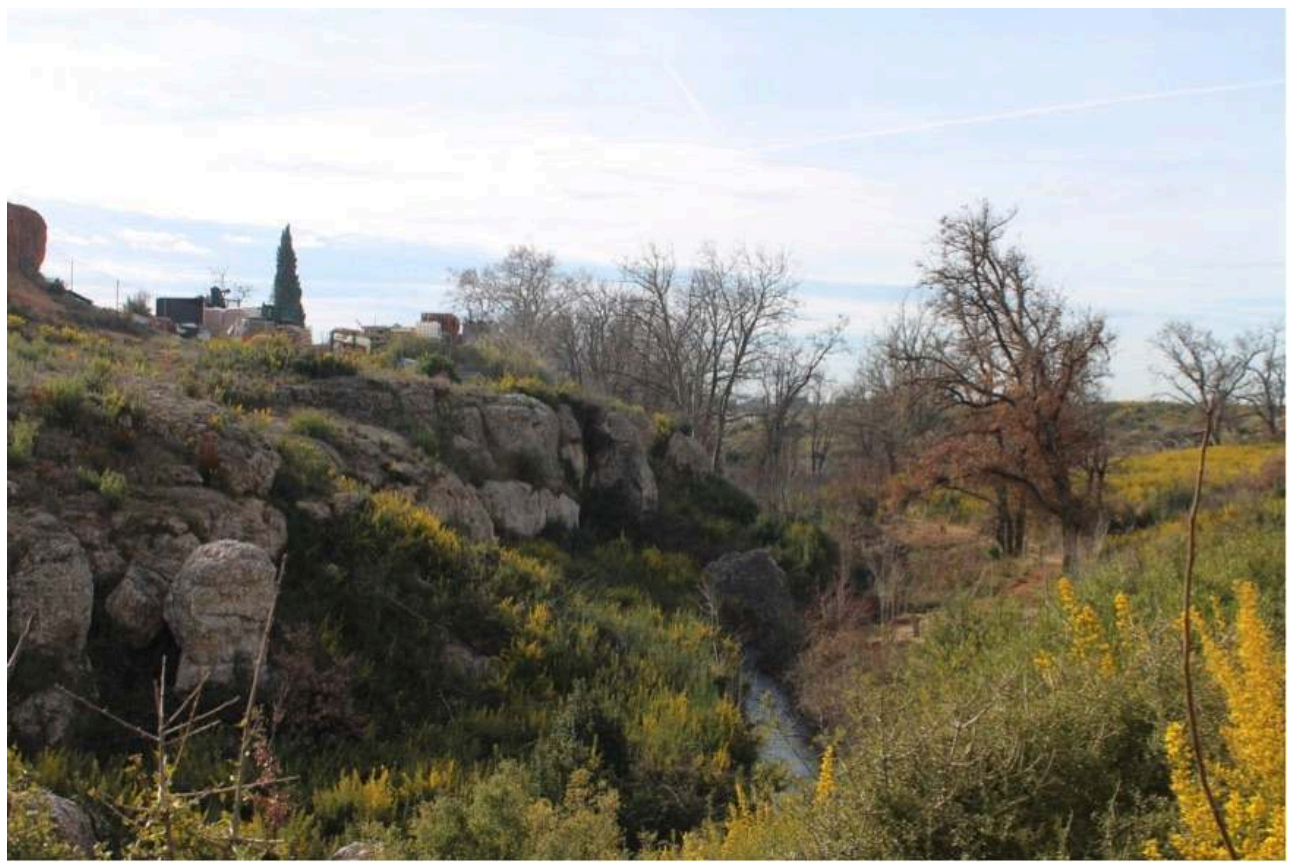

Source : Sylvie Salles, 2021.

Quarante ans plus tard, Michel Peraldi identifie, avec Michel Samson, des «lutteurs urbains dans la ville créative» qui gravitent dans des réseaux d'affinités professionnelles, " communautaires au sens villageois », autour d'un engagement dans la médiation culturelle (Peraldi et Samson 2020, p. 186-187). Le collectif des marcheurs ayant tracé le GR2013 en fait partie. Ils s'étaient croisés lors des «balades patrimoniales » que l'historienne Christine Breton organisait pour faire identifier leur patrimoine aux habitants des quartiers Nord de Marseille en lien au Grand Projet urbain $^{14}$. Au fil des marches, se sont agrégés artistes, universitaires, curieux, architectes, paysagistes, acteurs institutionnels, etc. Dans ces marches, pour elle :

«Les habitants sont des ambassadeurs, des relais. C'est la démultiplication qui crée

la surprise. Je pense que nous sommes en train de vivre un moment de grand

changement dans le travail culturel. Il y a un désir fort de partage et de prise en

charge. Je dis souvent que je préfère le mot tropos [50 attitude partagée ${ }_{20}^{[0}$ à celui de

topos 䂑 lieu. Hôtel du Nord est une attitude partagée par des habitants vis-à-vis d'un

territoire. » (Breton 2011, p. 10.)

La coopérative Hôtel du Nord, cofondée par Julie de Muer et des habitants en 2011, est née de ces balades pour développer un réseau de chambres chez l'habitant en lien au GR2013. Ici, le Sentier métropolitain est à la fois topos (lieu) et tropos (attitude partagée). Huit ans après Marseille-Provence 2013, deux initiatives animent toujours cette dynamique culturelle depuis le GR2013 : l'Observatoire photographique des paysages (OPP) depuis le GR2013 ${ }^{15}$ et le Bureau des Guides du GR2013 ${ }^{16}$.

\section{L'Observatoire photographique des paysages depuis le GR2013}

En 2012, année zéro du GR2013, Geoffroy Mathieu et Bertrand Stofleth ${ }^{17}$ ont proposé un projet artistique pour Marseille-Provence 2013, détournant les OPP « officiels » qui sont des outils de politique publique d'identification des évolutions des paysages ${ }^{18}$ (Mathieu et Stofleth, 2013 ; Mocquet, 2016). Leur OPP est toujours actif, avec les reconductions 
annuelles des 100 points de vue qu'ils ont choisis avec un "comité de pilotage sensible» issu de la communauté des marcheurs du GR2013. Le titre "Paysages usagés » met en avant la tension, entre violence et résistance, qu'ils ont trouvée dans la plupart des paysages traversés. Celle-ci vient du fait d'avoir côte à côte un acte d'aménagement qui fait violence au territoire, et des actes de résistance qui sont des manières de s'approprier un territoire improbable ou une inventivité déployée pour vivre avec. C'est un minuscule potager cultivé dans un " réduit foncier » laissé par Plan de Campagne, la plus grande zone commerciale périurbaine de France. Ce sont les reconstructions successives, quasiment au même endroit, d'un campement qui a été chassé en mettant une clôture, etc. Cette tension est finement installée par leur parti pris photographique descriptif d'observateur-arpenteur attentif à ce que le territoire révèle. Il n'y a pas d'effet de flou, ni de zoom afin de saisir tous les éléments contenus dans le cadrage. Les 100 points de vue, répartis sur l'ensemble du GR2013, sont un échantillonnage représentatif des paysages de la métropole, qui révèle aussi des enjeux récurrents de fragilité des milieux naturels, de vues emblématiques privatisées, de paysages pollués ou délaissés.

Figure 5. Étang de Bolmon (Châteauneuf-les-Martigues), 11 h 26 le 20 mars 2012

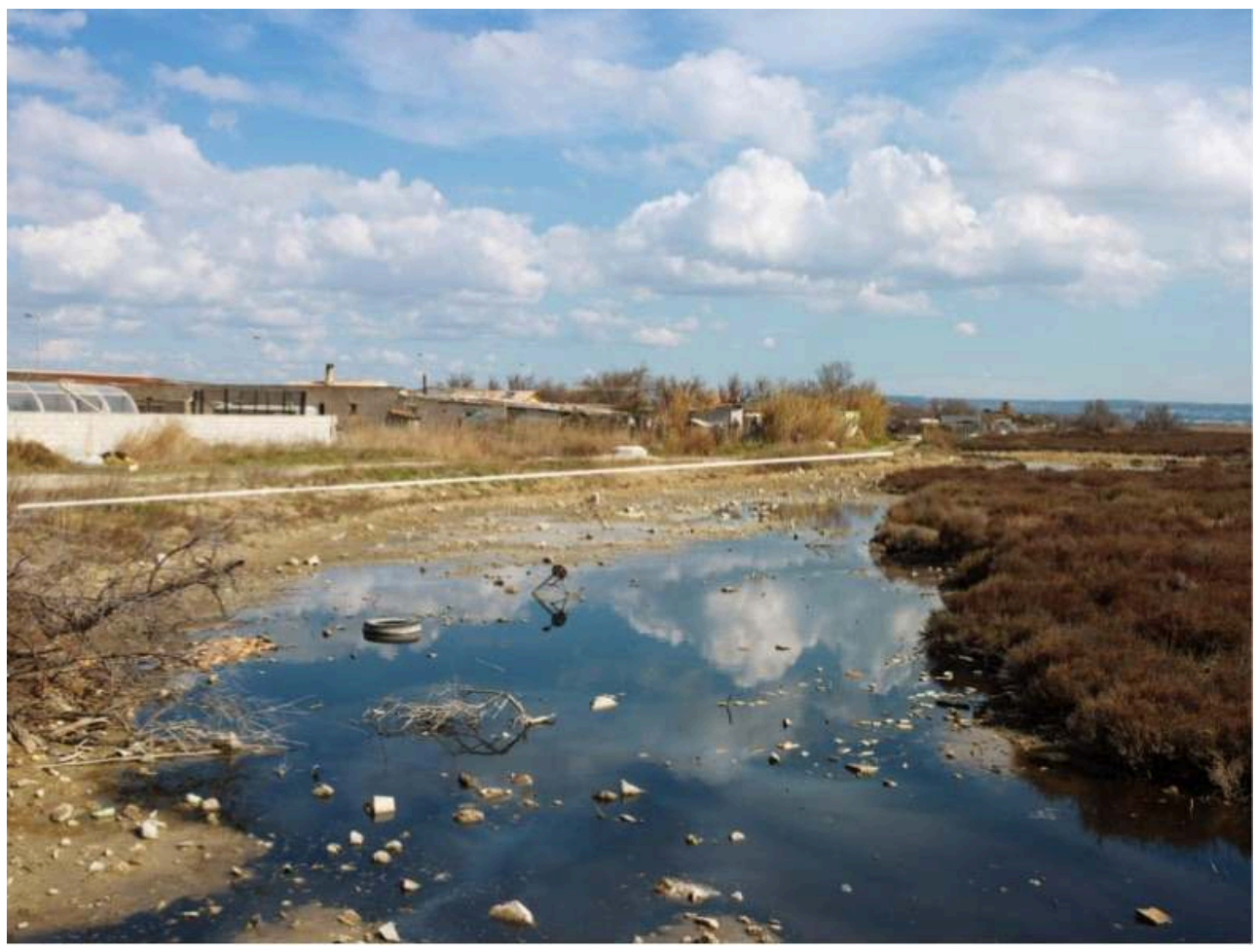

Source : Geoffroy Mathieu et Bertrand Stofleth

Pour faire les reconductions annuelles, ils ont recruté et formé aux techniques de la reconduction 70 adoptants, en installant leur caravane d'agents-recruteurs dans chacun des lieux de rendez-vous publics mensuels organisés durant l'année 2013. Depuis, l'animation de l'observatoire est un espace de convivialité, où une à deux fois par an les adoptants parlent de leur rapport au paysage. Si l'observatoire vit dans la durée, les adoptants eux-mêmes en questionnent la finalité et les résultats. Leurs questions ouvrent une démarche d'enquête sur les évolutions : leurs raisons, l'impact de travaux, la manière dont une action individualisée peut privatiser un paysage 
emblématique, à l'image d'une plaine agricole clôturée par une haie qui d'année en année ferme la vue sur la Sainte-Victoire. Les adoptants, comme les excursionnistes et les habitants des quartiers Nord, sont des passeurs. Chacune de leurs reconductions informe les processus de mutation des paysages contemporains et installe un espace de réflexion critique sur ces mutations.

\section{Le Bureau des guides du GR2013}

17 La démarche du Bureau des guides du GR2013 participe d'un engagement comparable, engagé avec le projet Caravan ${ }^{19}$ durant Marseille-Provence $2013^{20}$. Le CAUE des Bouches-du-Rhône a coproduit avec Radio Grenouille un voyage à pied d'un an pour découvrir les 38 communes traversées par le sentier qui formaient les «quartiers » de ce que Nicolas Mémain appelait un «hyper-village provençal " pour ne pas prononcer le mot métropole. Les marches étaient les étapes d'une série de lectures collectives, retransmises sur Radio Grenouille, où des élus, des architectes et des artistes racontaient le territoire à des habitants, visiteurs, techniciens territoriaux, etc. Le Bureau des guides du GR2013, créé en 2014 pour continuer à faire vivre le Sentier métropolitain, est né de cette idée que « le territoire peut se raconter à plusieurs et que de cette polyphonie émerge un savoir qui informe le geste de l'aménagement». Le GR2013 est le « rail » que suivent ces nouveaux guides pour enrichir la connaissance des territoires et des lieux traversés en donnant à entendre ce que chacun dit et pense. En accordant la même valeur à chaque récit, "l'école buissonnière poétique, joyeuse et insolente ", que décrit Alexandre Field, questionne le fait métropolitain avec l'idée que l'on n'hérite pas d'une métropole, mais qu'elle est le produit d'une inter-territorialité qui se construit à partir de la culture propre à chacun de ses « pays-paysages ».

Cette connaissance commence sur le terrain, dans les conversations marchées où les guides partent à la rencontre d'habitants, d'associations, d'élus locaux, d'institution métropolitaine, d'experts, de scientifiques, etc. Ils se définissent comme des « agents de liaison » mettant en présence différentes manières de dire, de regarder ou d'aménager le territoire et comme des " agents de conversation » autour de l'idée de poser les bases d'un accord pour penser le territoire - et l'aménager - autrement. Dans ce rôle, ils ont guidé la mission interministérielle en charge de mettre en place la métropole, face à qui Alexandre Field précise que «l'autorité du Bureau des guides était d'avoir fait le tour de la métropole à pied. C'était un fait, mais surtout une leçon d'humilité car avoir fait plusieurs fois le tour du GR2013 à pied nous permettait de dire à quel point nous connaissions mal la métropole ». Pour donner « un état de service de terrain » aux trois équipes (Devillers et associés, Lin et Seura architectes) retenues pour la consultation urbaine et métropolitaine, le Bureau des guides a organisé quatre randonnées métropolitaines entre deux gares, d'une zone commerciale à une autre, le long d'un chenal industriel et sur une voie ferrée abandonnée (Théry, 2016, p. 48-67). Ces marches, au moment où la création d'une métropole bousculait les organisations territoriales en place, ont contribué à réagencer les priorités politiques en mettant le paysage au centre des réflexions métropolitaines ${ }^{21}$. Mais le Bureau des guides y a aussi été un "agent de liaison" faisant remonter des appropriations et une culture métropolitaine bien plus complexes. Effectivement, comme les 100 points de vue de l'OPP «Paysages usagés », les marches qui suivent le fil du sentier GR2013 parlent de lieux, de gens, d'usages, de rencontres, d'histoires, etc., qui fabriquent aussi la métropole. 
Figure 6. Conversation marchée sur le GR2013, autour du Plan de paysage

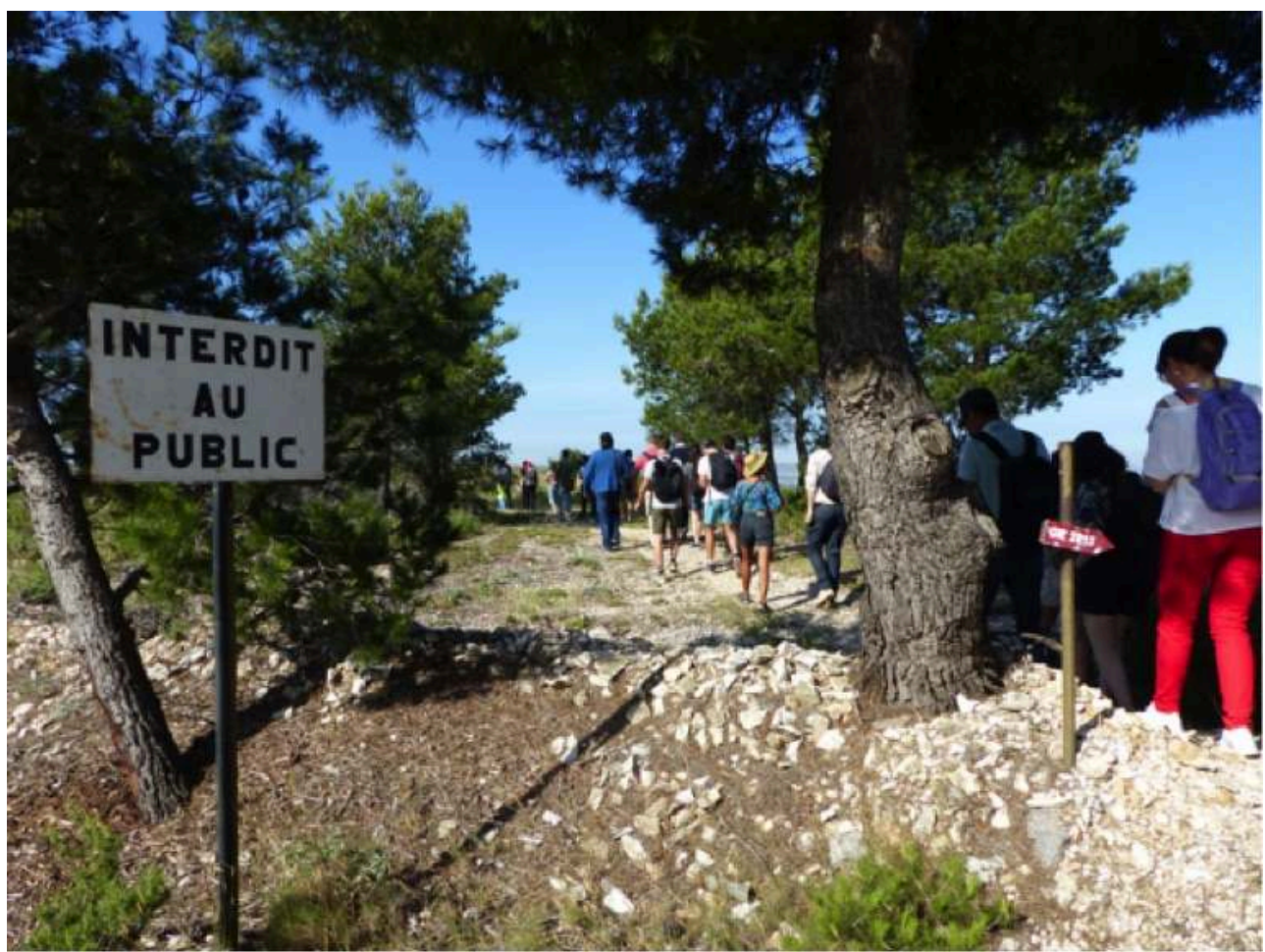

Source : Adèle Justin, ENSP, 2020.

\section{Vers un paysage horizon commun métropolitain}

Les modalités relationnelles, dont l'OPP et le Bureau des guides se font les intermédiaires depuis le GR2013, traduisent une connivence au territoire d'ordre " domestique ", au sens de Luc Boltanski et Laurent Thévenot, car elle s'inscrit dans des relations d'interdépendances dont les paysages sont les vecteurs. De ce point de vue, si le paysage incarne une dimension métropolitaine, ce n'est pas une question de dimension ni d'unité territoriale. Ce qui émerge de façon saillante avec ces deux projets, c'est «un principe supérieur commun » qui n'est pas «l'intérêt général, équitable pour tous mais pas forcément commun» (Boltanski et Thevenot, 1991). En effet, le parti pris descriptif des photographies de l'OPP et la polyphonie des récits relayés par le Bureau des guides véhiculent une connaissance qui part des lieux et des relations s'établissant entre des lieux et des hommes ou entre des hommes autour de lieux. La position transitionnelle des excurs, des habitants des quartiers Nord, des guides du GR2013 ou des adoptants de points de vue permet de faire circuler ces connaissances et de les diffuser, notamment pour informer l'action institutionnelle. À travers ces «agents » qui servent d'intermédiaires, l'attention aux milieux naturels unit les dimensions politiques et vernaculaires du paysage. Les projets portés par le GR2013 défendent tous l'idée d'une relation ville-nature dans laquelle les qualités vécues des paysages sont indissociables de la bonne fonctionnalité des écosystèmes naturels. Les "frottements ville-nature" que le sentier met en avant parlent de conditions d'habitabilité, dégradées ou non, dans lesquelles penser la métropole, c'est d'abord « revenir à ces notions fondamentales : traverser les paysages, habiter la pente, 
cheminer à l'ombre des ruisseaux, s'extraire de la ville dans un vallon, se retourner et observer la vallée depuis les hauteurs calcaires?» (ENSP et al., 2015, p.7). Si l'expérience in situ de la marche engage une cospatialité mettant en relation des lieux et des impressions (Chardonnet-Darmaillacq, 2016), elle facilite aussi la compréhension conjointe de dynamiques naturelles et anthropiques que les projets actuels du Bureau des guides convoquent plus clairement comme un horizon métropolitain commun.

\section{Le bassin versant des Aygalades, comme horizon vernaculaire et politique}

La remontée à pied du ruisseau des Aygalades ${ }^{22}$ en 2017 illustre cette capacité d'une pratique culturelle à négocier des interdépendances sociales, territoriales et environnementales. L'attention portée au ruisseau, avec la mission de conservation intégrée du patrimoine de Christine Breton, a progressivement fédéré des riverains et des associations. Les rendez-vous mensuels aux Aygalades ont débouché sur la création $\mathrm{du}$ collectif des Gammares ${ }^{23}$, réuni pour "prendre soin du ruisseau, favoriser un meilleur partage des connaissances, relier les initiatives et les territoires du bassin versant, proposer des actions communes ${ }^{24} »$. Ces actions communes parlent d'usages, de qualité des eaux, de biodiversité, de vision globale d'un cours d'eau oublié. Leur action va plus loin que la renaturation prévue dans le projet urbain d'Euroméditerranée ${ }^{25}$ en prenant en compte la continuité du cours d'eau dont la série photographique de Geoffroy Mathieu, intitulée La mauvaise réputation du ruisseau des Aygalades, reconstitue le fil.

«Un siècle d'urbanisation et d'industrialisation ont eu raison de sa continuité, de son débit et de son équilibre écologique à tel point que certains habitants en ont oublié même son existence. » (Mathieu, 2020.) 
Figure 7. Le soin des Aygalades par ses riverains

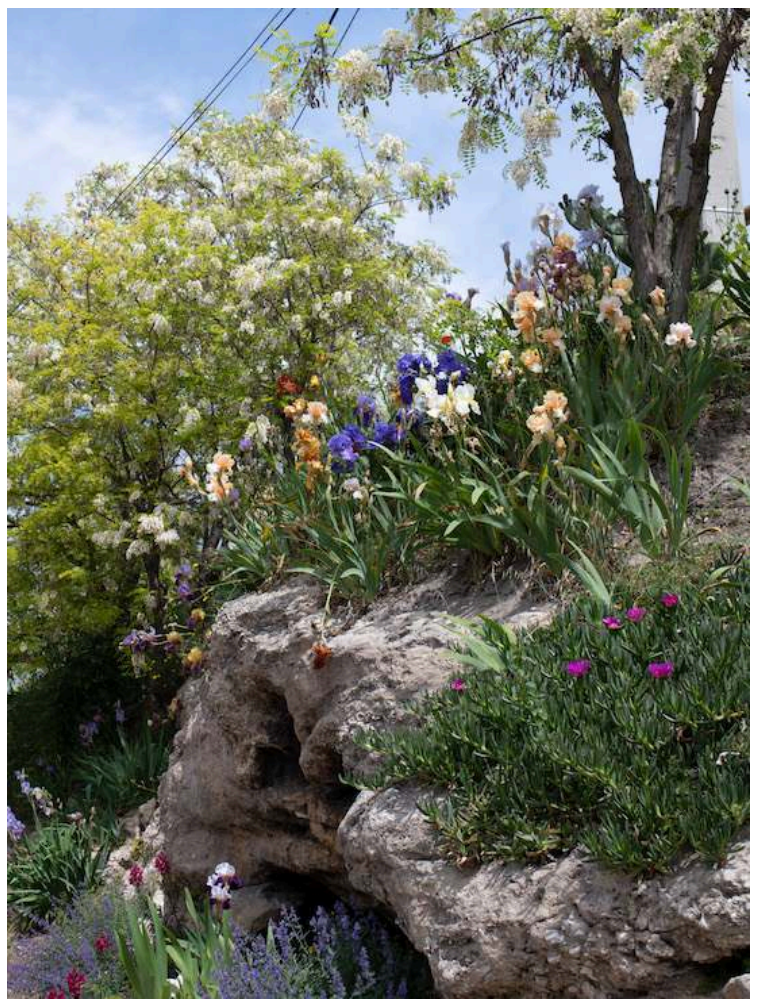

Source : Geoffroy Mathieu, 2020.

21 Depuis, la protection citoyenne proposée par le collectif des Gammares est plus qu'une gestion intégrée ${ }^{26}$ car elle renvoie au paysage de proximité des riverains, comme à l'ensemble du bassin versant. La gestion du paysage du cours d'eau et de la qualité écologique de l'eau et de ses rives pose les bases d'un horizon commun où le cours d'eau est un bien commun métropolitain, au sens où il croise des valeurs sociales, écologiques et territoriales qui font sens pour les riverains, comme pour les acteurs de la métropole. Cette gestion intégrée s'appuie sur des usages collectifs et festifs des berges façonnant des règles collectives (Ostrom 1980), qui garantissent une bonne gestion écologique du cours d'eau.

\section{À la recherche du paysage de l'étang de Berre}

L'expédition Pamparigouste ${ }^{27}$, à la recherche de l'étang de Berre, lancée en 2019 par le Bureau des guides, témoigne cette fois de la manière dont le paysage politique se modèle au contact du paysage vernaculaire. Pour Alexandre Field, cette expédition vers un pays imaginaire des Contes et Légendes de Provence dont le nom signifie "pétaouchnok» permet de se détacher de visions paradoxales ou conflictuelles. Le paysage de l'étang de Berre est patrimonial, rural, balnéaire, naturel, urbain, pollué, industriel... Il déstabilise autant qu'il fascine ou suscite de l'attachement. Si l'état de l'eau de la lagune est surveillé, le paysage des rives est le produit de choix d'aménagement et de conservation, liés à des décisions de l'État ayant atterri en rang dispersé sur le territoire de chaque commune. Michel Peraldi ${ }^{28}$ décrit le paysage de l'étang de Berre comme une énigme, impossible à caractériser parce qu'il est produit par un «bricolage interstitiel ». L'urbanisation et l'industrialisation ont poussé sur un 
terreau culturel et social riche en mémoires industrielles et locales, en terroirs agricoles, en milieux naturels. De fait son paysage ne devient lisible que par les différences que l'aménagement introduit ou par la manière dont celui-ci lézarde un état antérieur sans jamais totalement l'effacer. Le politique et le vernaculaire s'additionnent, dans des lieux où l'on accède souvent par surprise, parfois par effraction.

«Le récit des beautés de l'étang de Berre est d'abord récit d'expériences où [...] si toutes les pratiques sont loin d'être des braconnages, tous les pratiquants en revanche ont l'air de braconniers. » (Peraldi, 1989, p. 284.)

L'exploration Pamparigouste est une manière de rentrer par effraction pour faire entendre, à travers les récits des riverains, la complexité des cohabitations qui ont produit ce paysage hybride, vernaculaire et politique, mais surtout « culturel-naturelindustriel ». Au moment où cet équilibre est remis en question par la montée des eaux et les mutations industrielles, l'excursion à la recherche de l'étang de Berre conduite par le Bureau des guides engage une réinvention qui ne fait que commencer. La sociologue Christelle Gramaglia ${ }^{29}$ souligne que seule l'exploration permet la reconnaissance des forces de ce territoire, parmi lesquelles elle pointe l'aptitude des populations riveraines à défendre leur territoire (Gramaglia et Duperrex, 2021). De ce point de vue, le projet Pamparigouste fait remonter des capacités qu'ont des communautés à anticiper les changements de leurs milieux de vie et, en retour, des manières de l'aménager. Ici:

«Understanding environment involves recognizing that human life is lived as an integral part of a physical and cultural medium, under conditions through which people and places join together to achieve shape and identity. Within this environmental medium occur the activating forces of mind, eye, hand, climate, and the other processes of nature, along with the perceptual features and structural conditions that engage these forces and evoke their reactions ${ }^{30}$.» (Berleant, 1991, p. 102).

Cette expérience esthétique, reliée à des usages et à des objectifs, permet à des groupes d'envisager un horizon commun. 


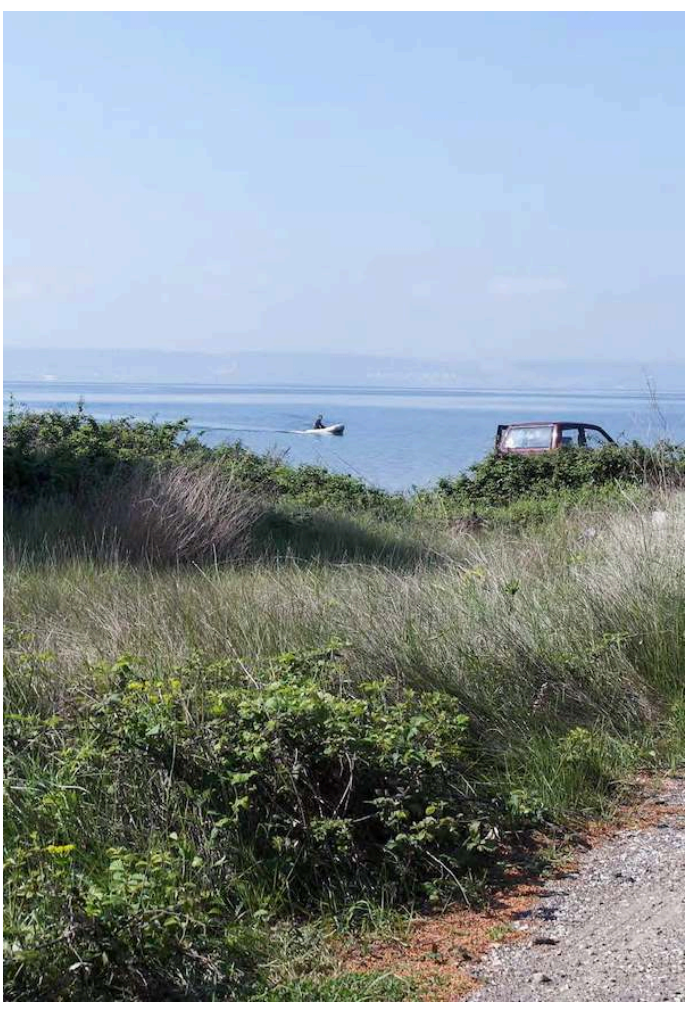

Source: Oriane Malanot, ENSP, 2017.

\section{Conclusion}

Les chemins de travers ouverts par le GR2013, aux confins d'espaces urbanisés, naturels ou agricoles, sont des fenêtres d'exploration dans les interstices du paysage métropolitain. Le Bureau des guides y a identifié des cohabitations entre des visions organisatrices, des usages et des fonctionnalités écologiques. Dans les «Paysages usagés ", de l'Observatoire photographique des paysages de Geoffroy Mathieu et Bertrand Stofleth, ces interstices sont les lieux d'une tension entre un paysage politique, faisant violence au territoire, et un paysage vernaculaire, témoignant de résistances pour vivre avec, qui se retrouvent dans les récits des habitants et usagers de l'étang de Berre. Tous ces projets culturels, qui ont pris le GR2013 comme véhicule, font découvrir «d'autres intelligibilités » (Jullien, 2012) en se mettant dans une situation d'intermédiaire. Celle-ci opère un écart ou une mise à distance (technique, scientifique ou fictionnelle) qui permet une réflexivité sur une réalité, perçue et vécue ici et maintenant, autour des expériences in situ que ces projets mettent en place et à partir desquelles il devient possible de penser un commun. Le long du GR2013, ces interstices $\mathrm{du}$ paysage sont concrets et renvoient aux «états-limites du paysage » que Michel Corajoud voit là où il $\mathrm{y}$ a « une sur-activation particulière, par la présence d'une substance étrangère » et où « il n'est pas étonnant que dans cette couche d'instabilité où deux milieux s'affrontent, la vie végétale puisse s'installer » (Corajoud 1982, p. 38). Les paysages usagés aux marges de l'urbanisation, le paysage oublié des Aygalades ou les paysages hybrides de l'étang de Berre ont une dynamique d'activation à la fois 
sociale et écologique. Cette dynamique est d'autant plus puissante qu'elle ouvre des horizons d'actions liées à la gestion comme à l'usage.

Cette attention aux interfaces, du côté de l'institution métropolitaine, est une clé d'entrée du plan de paysage métropolitain d'Aix-Marseille-Provence conduit par le paysagiste Bertrand Folléa. Pour qualifier ces interstices du paysage, il s'appuie sur la notion d'écotone, qui est une zone de transition écologique entre plusieurs écosystèmes. La figure de l'écart n'est pas convoquée, mais l'attention visuelle portée sur le paysage se déplace vers un point de vue écologique, économique, culturel et social qui élargit l'attention politique de la métropole sur le paysage, en le considérant comme « un monde à vivre dont nous sommes responsables » (Folléa, 2019). Le Bureau des guides du GR2013 et l'OPP " Paysages usagés » y contribuent, avec une mission d'animation confiée par la métropole. Pour autant, il y a un embarras à les intégrer dans un dialogue plus institutionnel. Là où le Bureau des guides est un «agent de conversation » qui fait entendre toutes les voix des lieux, l'animation du plan de paysage cherche à convoquer des coopérations autour d'actions pouvant être portées par les communes ou d'autres gestionnaires ${ }^{31}$. Le plan de paysage pose des enjeux transversaux - de risques, de dégradation du patrimoine naturel et construit ou d'attractivité et de confort de vie - qui sont des jalons de réciprocités entre les territoires. En revanche, dans ce dialogue ciblant plutôt des acteurs institutionnels, il ne convoque pas, du moins pour le moment, les compétences et les savoirs de ceux qui utilisent et vivent les paysages de la métropole, à l'image du collectif des Gammares qui est un acteur impliqué dans l'usage, la connaissance et la gestion du ruisseau des Aygalades.

Là, les intermédiaires culturels gravitant autour du GR2013 ont posé les bases d'une écologie qui mobilise toutes les formes de relation que l'homme entretient avec ce qui l'entoure. Ce sont aussi des bases pour que le paysage, dans sa dimension politique, ouvre des droits subjectifs qui ne procurent pas seulement des expériences, mais donnent aussi des "capabilités " (Sen, 2010), au sens d'aptitude à avoir prise sur des qualités d'existence dont les paysages sont les empreintes et les matrices. Il y a là un potentiel de glissement des modes de fabrication du paysage vernaculaire dans le champ du politique, et inversement. Ces projets artistiques et culturels activent des approches intégratives, qui convoquent sur un pied d'égalité les logiques biologiques, écologiques et humaines à l'œuvre dans nos environnements. Ils rendent visibles des interdépendances, où les dimensions, politique et vernaculaire, du paysage des périphéries contemporaines sont indissociables de la part vivante, au sens écologique et relationnel, des paysages.

\section{BIBLIOGRAPHIE}

Bédard, M., Le Paysage : un projet politique, Québec, éditions PUQ, 2009.

Berleant, A., Art and Engagement, Philadelphia, Temple University Press, 1991. 
Berque, A., Médiance. De milieux en paysages, Paris/Montpellier, Belin/GIP Reclus, coll. « Géographiques », 1990.

Bertho, R., La mission photographique de la Datar : un laboratoire du paysage contemporain, Paris, La Documentation française, 2013.

Besse, J.-M., Habiter un monde à mon image, Paris, Flammarion, 2013.

Boltanski, L., Thévenot, L., De la justification. Les économies de la grandeur, Paris, Gallimard, 1991.

Borruey, R., «L'architecture des territoires métropolisés... L'avenir métropolitain de Marseille », Conseil\#23, actes du séminaire des architectes-conseils de l'État, Marseille, mars 2018, p. 22-29.

Breton, C., « Dormir la tête au nord ? ", Esprit de Babel, n 4, 2011, p. 10.

Careri, F., Walkscapes. La marche comme pratique esthétique (2002), traduit de l'italien par J. Orsoni, Arles, Actes Sud, 2013.

Chalas, Y., « La ville de demain sera une ville-nature ", L'Observatoire, vol. 37, n² 2, 2010, p. 3-10.

Chardonnet-Darmaillacq, S. (dir.), Le Génie de la marche, Paris, éditions Hermann, 2016.

Corajoud, M., « Le paysage c'est l'endroit où le ciel et la terre se touchent », dans Dagognet,

F. (dir.), Mort du Paysage ?, Seyssel, Champ-Vallon, 1982.

Deleuze, G., Guattari, F., Mille Plateaux, Paris, éditions de Minuit, 1980.

Detraz, Y., Zone Sweet Zone : la marche comme projet urbain, Marseille, Éditions Wildproject, 2020.

Di Méo, G., « La métropolisation. Une clé de lecture de l'organisation contemporaine des espaces géographiques ", L’Information géographique, vol. 74, n³ 3, 2010, p. 23-38.

ENSP-ENSAM-IUAR, « Carnet d'études Ville nature », étude MIPM-AMP, Marseille, 2015.

Folléa, B., L'Archipel des métamorphoses : la transition par le paysage, Marseille, Éditions Parenthèses, 2019.

Gramaglia, C., Duperrex, M. (dir.), Fos - Étang de Berre, un littoral méditerranéen au cœur des enjeux environnementaux, Rives Méditerranéennes, $\mathrm{n}^{\circ}$ 61, 2020, mis en ligne en mars 2021, URL : https:// journals.openedition.org/rives/7680

Jackson, J. B., À la découverte du paysage vernaculaire (1984), Arles, Actes Sud, 2003.

Jullien, F., L'Écart et l'Entre. Leçon inaugurale de la Chaire sur l'altérité, Paris, éditions. Galilée, 2012. Lanaspèze, B., Lavessière, P.-H., L'Art des sentiers métropolitains, Paris, éditions du Pavillon de l'Arsenal 2020.

Lanaspèze, B., Marseille, ville sauvage : essai d'écologie urbaine (2012), Arles, Actes Sud, 2020.

Lavessière, P.-H., La Révolution de Paris. Sentier métropolitain, Marseille, Éditions Wildproject, 2014.

Mathieu, G., La Mauvaise Réputation, Marseille, éditions Zoème, 2020.

Mathieu, G., Stofleth, B., Paysages usagés : 100 points de vue depuis le GR2013, Marseille, Éditions Wilproject, 2013.

Mitchell, W.J.T., Landscape and Power (1994), University of Chicago Press, 2002.

Mocquet F., «L'Observatoire photographique national du paysage, transformations d'un modèle et hypothèses renouvelées de paysage ", Projets de paysage, $\mathrm{n}^{\circ} 15$, décembre 2016, URL : http:// journals.openedition.org/paysage/7099 ; DOI : https://doi.org/10.4000/paysage.7099 
Olwig, K.R., « Recovering the Substantive Nature of Landscape ", Annals of the Association of American Geographers, vol. 86, ${ }^{\circ}$ 4, December 1996, p. 630-653.

Ostrom, E., Governing the Commons, New York, Cambridge University Press, 1990.

Peraldi, M., Anselme, M., Jollivet, A., Boutron, J., Parisis, J.-L., Prono, M., Reboud, J.,, Paysage, ville et mémoire: Marseille, compositions et recompositions des paysages péri-urbains marseillais, le cas des friches urbaines et industrielles, Marseille, Cerfise, 1988.

Peraldi, M., Samson, M., Marseille en résistances. Fin de règnes et luttes urbaines, Paris, La Découverte, 2020.

Peraldi, M., «L'étang de Berre, interprétation d'un paysage métropolitain », Ethnologie française, vol. 19, n 3, 1989, p. 273-290.

Peraldi, M., Parisis, J.-L., « La ligne bleue des Alpilles », Recherches, n 45, septembre 1981, p. 15-52. Roger A., Le Court Traité du paysage, Paris, Gallimard, 1997.

Ronai, S., « Marseille : une métropole en mutation », Hérodote, n 135, 4e trim. 2009, p. 128-147.

Roncayolo, M, L'Imaginaire de Marseille (Marseille, CCI, 1990), Lyon, ENS Éditions, 2014.

Salvesen, B., New Topographics, Göttingen/Paris, Steidl/Interart, 2010.

Sen, A., L'Idée de justice, Paris, Seuil, 2010.

Sgard, A., « Le paysage dans l'action publique : du patrimoine au bien commun », Paysage et Développement durable, vol. 1, n² 2, septembre 2010, URL : http://journals.openedition.org/ developpementdurable/8565; DOI : https://doi.org/10.4000/developpementdurable.8565

Sieverts, T., Entre-ville : une lecture de la Zwischenstadt (1997), Marseille, Éditions Parenthèses, 2004.

Stalker, À travers les territoires actuels, Paris, éditions Jean-Michel Place, 2000.

Tamisier, C., «Étude de faisabilité d'un Institut du paysage méditerranéen : rapport de synthèse et d'activités 1990-1995 », DIREN Paca, 1995.

Théry, L. (dir.), La Métropole par le projet Aix-Marseille-Provence, Marseille, Éditions Parenthèses, 2016.

Tiberghien, G., « Hodologique », Carnets du paysage. Cheminements, $\mathrm{n}^{\circ} 11$, Arles/Versailles, Actes Sud/ENSP, 2004, p. 7-25.

\section{NOTES}

1. La création au $1^{\mathrm{er}}$ janvier 2016, inscrite dans la loi de modernisation de l'action publique territoriale et d'affirmation des métropoles (2014), a fusionné 92 communes et 6 intercommunalités.

2. La recherche de la Plateforme d'observation des projets et stratégies urbaines Aix-MarseilleProvence (2019-2022) est soutenue par le programme POPSU Métropoles et par la métropole AMP : https://popsu.archi.fr/projet/metropole-aix-marseille-provence. La recherche, « Le grand paysage comme ressource(s)», est coordonnée par l'ENSP (École nationale supérieure de paysage) et associe des chercheurs de l'ENSAM (École nationale supérieure d'architecture de Marseille) et de l'IUAR (Institut d'urbanisme et d'aménagement régional) d'Aix-Marseille universités.

3. «Le paysage peut être représenté par la peinture, le dessin ou la gravure, par la photographie, le film et le théâtre, par l'écriture, la parole, et probablement la musique et autre "image 
sonore". Cependant, avant toutes ces représentations secondaires, le paysage est lui-même un milieu physique et multi-sensoriel (terre, pierre, végétation, eau, ciel, son et silence, lumière et obscurité, etc.) dans lequel sont encodés le sens et les valeurs culturelles ; que ceux-ci résultent de la transformation physique d'un lieu par le jardinage ou l'architecture, ou qu'ils se trouvent en un lieu, pour ainsi dire, "par nature”.» (Traduit par Sylvie Salles.)

4. Ces relations interpersonnelles sont importantes car autour du GR2013 et de Baptiste Lanaspèze gravite une constellation d'acteurs qui sont les cofondateurs du Bureau des guides du GR2013 (Alexandre Field, Julie de Muer et Loïc Magnant) ou qui ont proposé des projets sur le sentier pour Marseille-Provence 2013: l'Observatoire photographique des paysages usagés (Geoffroy Mathieu et Bertrand Stofleth), Caravan (CAUE13, Radio Grenouille et Wildproject), Promenades sonores (Radio Grenouille), Tentative d'encyclopédie marchée des Bouches-duRhône (Nicolas Mémain), Paysages gustatifs (collectif SAF), Agence Touriste (Mathias Poisson et Virginie Thomas), Hôtel du Nord, Trailpoints (Bryan Connell).

5. «New Topographics: Photographs of a Man-Altered Landscape» est une exposition photographique, organisée en 1975 par William Jenkins à la George Eastman House (Rochester), qui marque un tournant pour la photographie documentaire et la représentation des paysages, avec un point de vue détaché et neutre sur les paysages américains contemporains.

6. La mission photographique de la Délégation interministérielle à l'aménagement du territoire et à l'attractivité régionale est une commande publique passée à des photographes pour « représenter le paysage français des années 1980 ». La mission était dirigée par François Hers et Bernard Latarjet, qui était directeur de l'association Marseille-Provence 2013.

7. Stalker a été créé à Rome en 1995 par des étudiants architectes (Francesco Careri, Aldo Innocenzi, Paolo Bruschi, Giovanna Ripepi, Romolo Ottaviani, Valerio Romito et Lorenzo Romito). En 1993, ils avaient, durant 5 jours, exploré à pied les interstices, marges, espaces abandonnés ou en transformation de l'aire urbaine de Rome (http://www.osservatorionomade.net/tarkowsky/ manifesto/manifest.htm).

8. L'association Bruit du frigo a été créée en 1997 par ces deux étudiants en architecture, qui avaient investi la rue pour y chercher ce qu'ils ne trouvaient pas à l'école: une culture du quotidien rendant compte des réalités urbaines et sociales des lieux et des gens (https:// bruitdufrigo.com/)

9. L'architecte Denis Moreau a créé ce blog en 1995 où il rend compte de ses promenades dans les périphéries de Paris, pour construire une métropole imaginaire: https:// artdeboutblog.wordpress.com/2019/06/11/banlieue-de-paris-par-denis-moreau-en-cours/, consulté le 30-12-2020.

10. Le géographe-urbaniste Paul-Hervé Lavessière, qui a travaillé au Bureau des guides du GR2013, a raconté son voyage dans le Grand Paris dans La Révolution de Paris (2014), tandis que l'urbaniste Jens Denissen a cofondé en 2014 le collectif Le voyage métropolitain.

11. https://www.lesentierdugrandparis.com/le-projet

12. Le balisage programmé jusqu'en 2024 se fera dans le cadre d'ateliers des territoires portés localement par différents partenaires institutionnels. Ce sont des dispositifs de pilotage et d'animation pour faire émerger une stratégie de territoire et engager les acteurs locaux dans un processus de projet. «Comment les sentiers fabriquent nos villes?», débat animé par Baptiste Lanaspèze, Paris, Pavillon de l'Arsenal, $1^{\text {er }}$ octobre 2020 (https://www.pavillon-arsenal.com/fr/ arsenal-tv/conferences/hors-cycle/11865-comment-les-sentiers-fabriquent-nos-villes.html).

13. Le Cerfise a été créé à Marseille en 1975, par Michel Anselme et Michel Peraldi, en tant qu'antenne du Cerfi fondé par Félix Guattari en 1967.

14. Christine Breton a été conservatrice du patrimoine de la ville de Marseille, à partir de 1996, dans le cadre d'une mission expérimentale de patrimoine intégré qui était le volet culturel associé à la mutation industrielle et urbaine de la vallée du Séon.

15. http://www.opp-gr2013.com 
16. https://bureaudesguides-gr2013.fr/

17. Les propos qui suivent s'appuient sur des entretiens avec Geoffroy Mathieu et Bertrand Stofleth, le 28 octobre 2020 et le 4 novembre 2020.

18. Les Observatoires photographiques du paysage, mis en place à l'initiative de l'État en 1991 pour analyser sur le temps long les transformations des paysages, sont maintenant engagés à l'initiative de PNR, grands sites ou collectivités territoriales.

19. https://bureaudesguides-gr2013.fr/caravan/

20. Les propos qui suivent s'appuient sur un entretien réalisé le 6 novembre 2020 avec Alexandre Field, cofondateur du Bureau des guides, qui travaillait alors au CAUE 13.

21. Entretiens avec Vincent Fouchier (directeur général adjoint chargé du projet métropolitain AMP Métropole) le 10 novembre 2020 et avec Marie Baduel, directrice de la consultation, le 28 octobre 2020

22. Le ruisseau des Aygalades est un fleuve côtier, du massif de l'Étoile au quai d'Arenc (port de Marseille), dont le lit, busé et canalisé, est invisible ou inaccessible sur la majorité de son cours, sauf lors de ses crues.

23. Le collectif, créé en 2019, réunit le Bureau des guides du GR2013, Hôtel du Nord, l'Association pour la cité des arts de la rue (ApCAR), les CIQ riverains, l'Association des amis des Aygalades (AAA), l'AESE (Action Environnement Septèmes et environs), les artistes-voisins, Sud Side et le collectif SAFI.

24. https://bureaudesguides-gr2013.fr/les-aygalades/

25. Le projet urbain, confié à l'agence Leclercq Associés, programme pour l'Opération d'intérêt national Euroméditerranée un parc urbain où la réouverture du cours d'eau et sa renaturation visent à réguler les crues et les pollutions.

26. La gestion intégrée, appliquée aux cours d'eau, renvoie à une démarche participative ayant pour objectif de définir un équilibre entre les différentes fonctions du milieu et des usages de l'eau, ainsi que les actions à mettre en œuvre pour atteindre et maintenir cet équilibre.

27. https://bureaudesguides-gr2013.fr/pamparigouste/

28. Michel Peraldi a réalisé, entre 1952 et 1985, pour le secrétariat d'État chargé de l'Environnement, un inventaire des espaces naturels restant autour de l'étang de Berre après 30 ans de développement industriel et urbain, en lien aux recherches du Cerfise sur le statut des espaces naturels dans les sociétés contemporaines.

29. Conversation marchée Explorer \#02, le samedi 22 juin, avec Christelle Gramalia et Matthieu Duperrex le long du chenal de Caronte, organisée par le Bureau des guides du GR2013 en partenariat avec la ville de Martigues, Opéra Mundi et Radio Grenouille.

30. «L'environnement, alors, n'est pas un territoire étranger autour de soi. La compréhension de l'environnement implique de reconnaître que la vie humaine est vécue comme une partie intégrante d'un médium physique et culturel, dans lequel les gens et les endroits, ensemble, constituent une forme et une identité. Dans cet environnement, l'esprit, l'œil, la main, le climat et les autres processus naturels sont des forces d'activation, avec des caractéristiques perceptives et des conditions structurelles qui engagent ces forces et évoquent des réactions. » (Traduit par Sylvie Salles.)

31. Entretien avec Marc Del Corso et Florence Hannin, du service Paysage de Aix-MarseilleProvence Métropole, le 6 novembre 2020. 


\section{RÉSUMÉS}

Cet article analyse le rôle du sentier métropolitain GR2013, créé pour Marseille-Provence capitale européenne 2013 de la culture, en relation à la mise en place d'une métropole institutionnelle. Les randonnées et projets artistiques programmés sur le sentier, aux marges des périphéries urbanisées, ont donné à voir les paysages de la métropolisation. Si ce sont des paysages vernaculaires dont les appropriations semblent résister au milieu du paysage politique des grands aménagements, la dynamique associative du GR2013 a fait émerger d'autres intelligibilités en relayant des récits de riverains. Au fil des randonnées, des paysages hybrides articulent des dimensions vernaculaires et politiques, distinguées par John Brinckerhoff Jackson. Autour du GR2013, le sens du paysage politique s'élargit d'aptitudes collectives à gérer des interdépendances sociales, fonctionnelles et écologiques. Cette intégration s'opère là où les paysages vernaculaires et politiques entrent en contact, dans des interstices que le plan de paysage métropolitain saisit en matière d'écotones. C'est un pas vers la prise en compte de la part politique des paysages vernaculaires contemporains.

This article examines the role of the metropolitan GR2013 trail created for the MarseilleProvence European Capital of Culture in 2013 in connection with the establishment of an institutional metropolis. The hikes and artistic events programmed along the trail, which skirts the edges of the urban peripheries, showcased the metropolitan landscapes. Despite these vernacular landscapes, the forms of appropriation of which seem to resist the political landscapes of major developments, the associative dynamics of the GR2013 have brought to light other intelligibilities by relating the stories of local residents. Along the way, hybrid landscapes express vernacular and political dimensions, as defined by John Brinckerhoff. Jackson. Around the GR2013 trail, the meaning of political landscape extends to include the collective capacity to manage social, functional and ecological interdependencies. This integration occurs where vernacular and political landscapes meet in the intervening spaces captured by the urban landscape plan in the form of ecotones. It constitutes a step towards considering the political dimension of contemporary vernacular landscapes.

\section{INDEX}

Mots-clés : paysage métropolitain, paysage politique, relation métropole-nature, randonnée métropolitaine

Keywords : metropolitan landscape, political landscape, metropolis-nature relationship, metropolitan trail

\section{AUTEUR}

\section{SYLVIE SALLES}

Sylvie Salles est architecte, docteure en études urbaines et professeure HDR en projet de paysage à l'École nationale supérieure du paysage de Versailles. Ses recherches au Larep portent sur les relations paysage/environnement et sur leurs rôles dans la transition écologique de l'urbanisme. s.salles[at]ecole-paysage[dot]fr 